\title{
Pre-filled Pen for Anti-calcitonin Gene-related Peptide Migraine Therapy
}

\author{
An Expert Interview with Rebecca Stuckey \\ University Hospitals Plymouth NHS Trust, Derriford Hospital, Plymouth, UK
}

DOI: https://doi.org/10.17925/ENR.2020.15.1.11

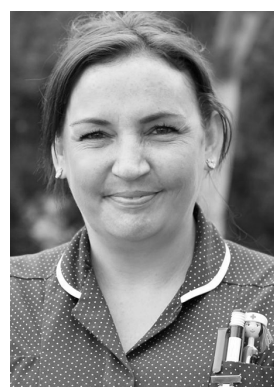

\section{Rebecca Stuckey}

Rebecca has been a senior clinical nurse specialist for 8 years, the past 5 years focusing in headache. She has found it to be the most exciting role she has been a part of in her whole career. Patients with headache disorders have historically been misunderstood, misdiagnosed and mistreated by the public, as well as in the medical profession. Rebecca enjoys empowering patients and their families with education and understanding. The idea that there are going to be so many treatment options available for her patients over the next few years is exciting; she states "It will change the way we work and hand back power to the individual. We are on the cusp of re-writing the rule book - I cannot wait to be part of it!"

\section{Keywords}

Calcitonin gene-related peptide, fremanezumab, migraine

Disclosure: Rebecca Stuckey reports acting as an occasional external expert spokesperson for Teva in the field of migraine.

Acknowledgement: Medical writing assistance was provided by Katrina Mountfort of Touch Medical Media and supported by Touch Medical Media.

Review Process: This is an expert interview and, as such, has not undergone the journal's standard peer-review process.

Compliance with Ethics: This is an expert interview and does not report on new clinical data, or any studies with human or animal subjects performed by the author.

Authorship: The named author meets the International Committee of Medical Journal Editors (ICMJE) criteria for authorship of this manuscript, takes responsibility for the integrity of the work as a whole and has given final approval to the version to be published.

Access: This article is freely accessible at touchNEUROLOGY.com ( ) Touch Medical Media 2020

Received: 28 July 2020

Published Online: 20 October 2020

Citation: European Neurological Review. 2020;15(1):11-2 Corresponding Author: Rebecca Stuckey,

Teva UK Limited, Ridings Point, Whistler Drive, Castleford, WF10 5HX, UK.

E: general.enquiries@tevauk.com

Twitter: @TevaUK

LinkedIn: www.linkedin.com/company/

teva-pharmaceuticals/

Support: No funding was received in

the publication of this article.
D espite the fact that migraine is the most prevalent neurological disorder worldwide, it remains under-diagnosed and under-treated in more than half of all patients. ${ }^{1}$ In the past decade, the development of monoclonal antibodies capable of blocking either calcitonin gene-related peptide (CGRP) or its receptor have transformed the management of migraine. ${ }^{2}$ At present, fremanezumab (Ajovy ${ }^{\circledR}$ ) is the only anti-CGRP drug recommended for use on the National Health Service in England and Wales by the National Institute for Health and Care Excellence (NICE) for chronic migraine patients. ${ }^{3}$

The latest advance in CGRP therapy has been the announcement that a pre-filled pen for fremanezumab injection has become available in the UK for the prevention of migraine in adults who have at least 4 migraine days per month. ${ }^{4}$ In an expert interview, Rebecca Stuckey, a specialist neurology nurse, discusses the burden of migraine and the likely impact of this innovation.

\section{Q. Could you please briefly tell us about the role of the headache nurse specialist in the care of patients with migraine?}

My role as a headache nurse specialist is broad and difficult to describe in a short paragraph. I support my patients and their families from the moment they are referred to the service with their diagnosis, treatment and symptom management. I advocate for them with employees and sickness tribunals. I do a lot of Botox (botulinum toxin) injections and nerve blocks. I run the drug trials and have currently got 164 patients about to commence fremanezumbab in September.

\section{Q. From a nursing point of view, what are the major unmet needs in the management of migraine?}

Migraine sufferers are the largest cohort of patients whom, as clinicians, we have historically failed to successfully treat; they have been misdiagnosed, misunderstood and mistreated with the wrong drugs for centuries. This is without mentioning the public misconceptions about them "just having a headache" and migraine being seen as an excuse rather than a real illness, meaning that predominantly working-age women are socially isolated and usually depressed, feeling a lack of any control over their lives. They lose the best years of their lives, whereby they should be attending university, excelling in the workplace or enjoying their children. The main reason for this has been a lack of available treatments. We have used a range of drugs that are used for high blood pressure, depression and seizures, which have been hard to tolerate and brought with them unwelcome side effects. Newer treatments such as Botox have changed the treatment landscape as it is far more effective, but painful, with over 30 injection sites, and heavily reliant on service provision. There are very few headache services in chronic migraine in comparison with any other specialties, therefore migraine sufferers continue to have a large unmet need. 


\section{Q. What has been the impact of anti-CGRP migraine therapy?}

The prospect of using anti-CGRP drugs is hugely important to us, as it is the first preventative specifically aimed at migraine. Also, it means that we now have options. Until now, if you didn't respond to Botox, that was it; there were no other options.

\section{Q. Could you give us an overview of the new pre-filled pen for fremanezumab injection?}

The prospect of using the new fremanezumab pre-filled pen gives power back to the individual, as they do not have to travel miles to see me. They can inject at home once a month and I can see their diaries electronically and review them over the phone. The injection pen is very easy to use and only takes a few seconds to administer.

\section{Q. What impact is this likely to have on patients,} as well as headache/migraine specialist clinics?

Moving forward, this will change the way we as clinicians work, with fewer face-to-face clinics and therefore, more efficient, productive ways to see more patients, either virtually or over the phone. For patients, it puts them back in control of where and when they have treatment; they do not have to be dependent on whether I am fully booked or on leave, therefore avoiding potential delays in receiving treatment. $\square$

GBD 2016 Headache Collaborators. Global, regional, and national burden of migraine and tension-type headache, 1990-2016: a systematic analysis for the Global Burden of Disease Study 2016. Lancet Neurol. 2018:17:954-76

Scuteri D, Adornetto A, Rombolà L, et al. New trends in migraine pharmacology: targeting calcitonin gene-related peptide (CGRP) with monoclonal antibodies. Front Pharmacol. 2019;10:363. National Institute for Health and Care Excellence (NICE). Fremanezumab for preventing migraine. Technology appraisal guidance [TA631]. 2020. Available at: www.nice.org. uk/guidance/ta631 (accessed 28 July 2020).

4. Teva UK Limited press release. Teva launches pre-filled pen for anti-CGRP migraine therapy AJOVY (fremanezumab) in the UK. 2020. Available at: www.acnr.co.uk/2020/08/cgrp-migraine/ (accessed 28 July 2020). 